\title{
Cross-phase modulation compensation in multichannel system based on filter bank multicarrier modulation
}

\author{
Oussama Gharbi $\odot,{ }^{\mathrm{a}, \mathrm{b}, *}$ Sofien Mhatli $\odot,{ }^{\mathrm{a}}$ and Rabah Attia ${ }^{\mathrm{a}}$ \\ ${ }^{a}$ Carthage University, Tunisia Polytechnic School, SERCOM Laboratory, Tunis, Tunisia \\ ${ }^{b}$ Institut Supérieur d'Informatique (ISI) du Kef- Université de Jendouba, Jendouba, Tunisia
}

\begin{abstract}
Futuristic mobile data networks are expected to reach higher data rates per user to accommodate the specifications of emerging services, such as triple play and mobile applications. Researchers are carrying out their works to tackle the drawbacks of traditional orthogonal frequency-division multiplexing by designing new waveforms with high spectral efficiency and low out-of-band emissions. Among many others, filter bank multicarrier (FBMC) is an appealing candidate for beyond $5 \mathrm{G}$ that fulfills these constraints. Consequently, it is worth to shed light on the performance of FBMC in multichannel transmission system. The main contribution of our paper is to compensate fiber nonlinearity including self-phase modulation and cross-phase modulation effects using adaptive Volterra equalizer in longhaul FBMC system based on a $6 \times 20 \mathrm{~Gb} / \mathrm{s}$ in wavelength-division multiplexing scheme over $100 \mathrm{~km} \times 30$ spans of singlemode fiber (SMF). The impacts of channel spacing, bit rate, and fiber types on system performance are addressed. For a target of bit error rate $=10^{-3}$, the simulations show that this performance can be reached at $-12 \mathrm{dBm}$ of launch power when 5-taps third-order Volterra (TOV) equalizer is used for $12.5 \mathrm{GHz}$ of channel spacing and an improvement of approximately $1 \mathrm{~dB}$ is obtained for 7 taps compared with the same equalizer with 3 taps. To further enhance the system performance, standard-SMF is replaced by a new class fiber called pure-silica-core fiber with large effective area, which exhibits a gain of almost 1.3 and $1 \mathrm{~dB}$ for 3 and 5 taps, respectively. The complexity burden of the TOV filter is also discussed. (C) The Authors. Published by SPIE under a Creative Commons Attribution 4.0 International License. Distribution or reproduction of this work in whole or in part requires full attribution of the original publication, including its DOI. [DOI: 10.1117/1.OE .60.9.095101]
\end{abstract}

Keywords: 5G networks and beyond; bit error rate; channel spacing; cross-phase modulation; filter bank multicarrier; Volterra filter; wavelength-division multiplexing.

Paper 20210469 received May 4, 2021; accepted for publication Aug. 19, 2021; published online Sep. 3, 2021.

\section{Introduction}

High-speed optical communications are applied in fronthaul, backhaul, and longhaul applications. With the appearance of $5 \mathrm{G}$ networks, the demand for new services, such as triple play, video on demand as well as mobile applications, becomes huge in terms of data rates and quality of services. Indeed, researchers nowadays are working on fronthaul applications and last meter networks based on different techniques, such as visible light communications technology, which is considered as one of the most attractive techniques for beyond 5G (B5G) wireless communications. ${ }^{1}$ In Refs. 2 and 3, authors experimentally demonstrate the use of free-space optics (FSO) techniques and millimeter-wave to enhance the reliability of 5G fronthaul part. Other researchers focus on the backhaul architecture of the networks; in Ref. 4, authors optimize the Capital Expenses of 5G backhaul with an FSO link.

For longhaul applications, wavelength-division multiplexing (WDM) is a promising solution for next-generation optical communications which allows a high capacity of data networks and a good spectral efficiency. ${ }^{5,6}$

Today, it is a well-known fact that $5 \mathrm{G}$ new radio (NR) wireless communications are based on orthogonal frequency-division multiplexing (OFDM) waveform. ${ }^{7}$ However, the main limitation of OFDM modulation is its high out-of-band (OOB) emissions, which poses a big challenge for

*Address all correspondence to Oussama Gharbi, oussama_gharbi@yahoo.com 
multiband systems and cognitive radio (CR). This leads to an inherent interference between adjacent frequency bands. For this purpose, OFDM modulation is substituted by a new competitive waveform for B5G with very low OOB level, known as filter bank multicarrier (FBMC) waveform. ${ }^{8}$ Furthermore, significant nonlinear effects are very pronounced for WDM system, namely, self-phase modulation (SPM), cross-phase modulation (XPM), and four-wave mixing (FWM). The primary goal of this paper is to propose a scheme based on FBMC in WDM link for B5G and assess the impact of the SPM and XPM, whereas the effects of FWM are ignored. Generally, several approaches have been proposed to dodge the nonlinear impairments of the channel according to transmission level. Some techniques are used at the transmitter side, such as channel coding (i.e., low-density parity-check codes ${ }^{9}$ and polar $\operatorname{codes}^{10}$ ). Some other solutions are applied in the fiber optical channel by implementing optical phase conjugation method $^{11}$ or deploying new fibers with a large effective area. ${ }^{12}$ At the receiver side, nonlinear effects can be mitigated by performing adaptive equalization. ${ }^{13}$

To compensate SPM and XPM impairments in WDM system, we use the two latter solutions, namely, digital equalization-based Volterra series and single-mode fiber (SMF) with large effective area.

This paper is organized as follows. Section 2 gives an overview of FBMC waveform, Sec. 3 describes the SPM and XPM computation method, Sec. 4 is devoted to the post-compensation technique based on Volterra equalizer, Sec. 5 describes the system setup, and simulation results are discussed in Sec. 6. Section 7 analyzes the complexity burden of Volterra and conclusions are drawn in Sec. 8.

\section{Filter Bank Multicarrier Technical Description}

In contrast to other modulation schemes, FBMC can comply with the Balian-Low theorem, ${ }^{14}$ which is based on three requirements: (i) good localization in both time and frequency domains, (ii) high spectral efficiency, and (iii) orthogonality in complex domain.

In the first of these three constraints, various pulse shape filters are proposed for FBMC modulation instead of the rectangular window used in traditional OFDM. The most known filters are PHYsical layer for DYnamic AccesS and cognitive radio (PHYDYAS), Root Raised Cosine, and Hermite filters. According to Fig. 1, the PHYDYAS filter experiences a good localization in both time and frequency domains, which can significantly reduce the inter symbols interference (ISI) and inter-channel interference (ICI), respectively.

The design of the PHYDYAS filter is based on frequency sampling technique ${ }^{15}$ by interpolating the designed frequency coefficients of the prototype filter, and the impulse response is obtained by performing the inverse Fourier transform which yields

$$
p[n]=1+2 \sum_{k=1}^{K-1}(-1)^{k} P_{k} \cos \left(\frac{2 \pi k}{N K} m\right), \quad m=1, . ., K N-1,
$$

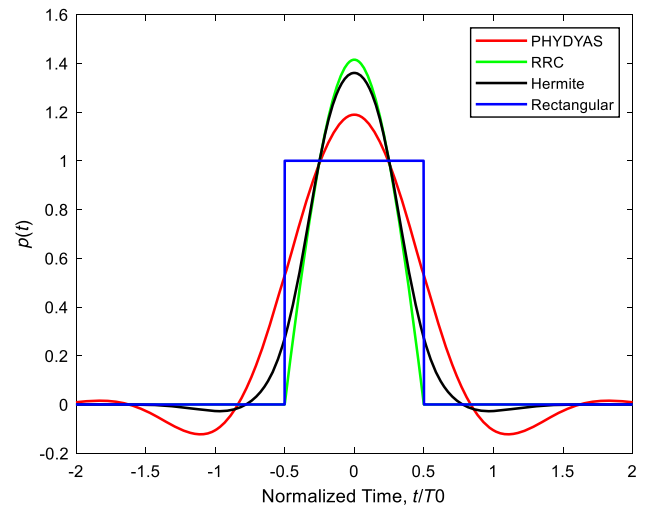

(a)

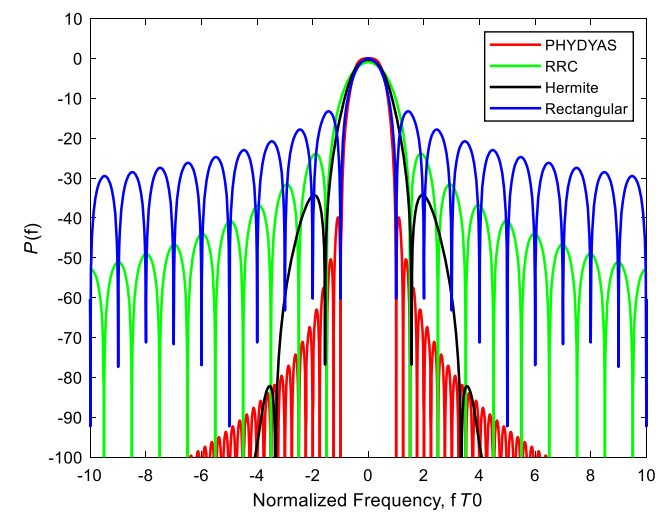

(b)

Fig. 1 Impulse (a) and frequency (b) response comparison of different filters. 


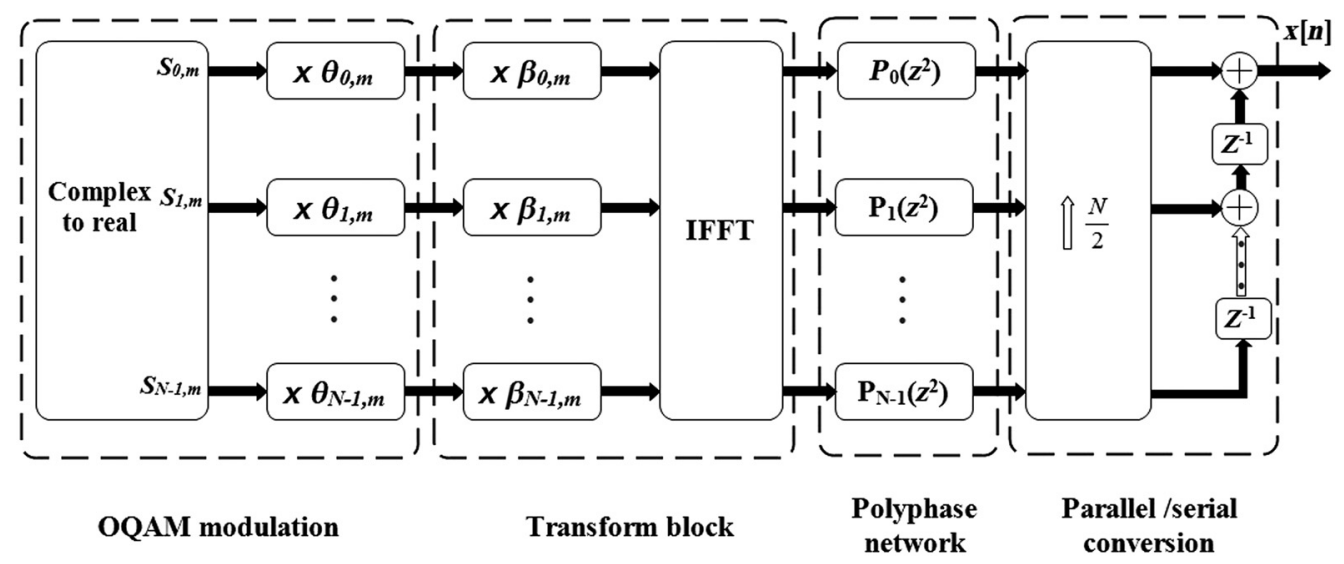

Fig. 2 PPN-FBMC/OQAM transmitter architecture.

where $K$ is the overlapping factor which commonly sets to its optimum value, $4 . P_{ \pm k}$ are the frequency coefficients of the filter which are in Ref. 16 as $P_{0}=1, P_{ \pm 1}=0.97196$, $P_{ \pm 2}=\sqrt{2} / 2$, and $P_{ \pm 3}=0.235147$.

Moreover, the used prototype filter is spread out in the frequency domain (equal to $N K$ ), which makes FBMC more robust against multipath fading (i.e., dispersion in optical communication). For this reason, the cyclic prefix is abandoned and the second condition of Balian-Low theorem is satisfied.

To circumvent the third constraint, the orthogonality among adjacent subcarriers is ensured by the so-called offset-QAM (OQAM), where the real and imaginary parts of the complex symbols occupy different time slots. It is important to point out that this orthogonality is restricted to the real domain (i.e., either real or imaginary part).

The discrete-time baseband signal $x[n]$ at the output of the proposed FBMC/OQAM transmitter can be written as ${ }^{17}$

$$
x[n]=\sum_{k} \sum_{m=-\infty}^{+\infty} s_{k, m} \theta_{k, m} \beta_{k, m} p(n-m N / 2) e^{j\left(\frac{2 \pi}{N}\right) k n},
$$

where $m$ is the sample at the output of OQAM process, $S_{k, m}$ is the real-valued data at subcarrier $k, \theta_{k, m}=j^{(k+m)}$ is used to maintain orthogonality between adjacent subcarriers, $\beta_{k, m}=$ $(-1)^{k m} \cdot \exp ^{(-j k(K N-1) \pi / N)}, N$ is the number of subcarriers, and $p$ is the prototype filter of length $N K$.

To reduce the complexity burden, Bellanger et al. proposed a new implementation called polyphase network (PPN). The basic idea is to decompose the prototype filter of length $N K$ into $N$ elementary filters, where each one has $K$ taps. The structure of PPN-FBMC based on OQAM modulation (PPN-FBMC/OQAM) is illustrated in Fig. 2.

In Fig. 2, $P_{i}\left(z^{2}\right)$ is the $i$ 'th polyphase component of the prototype filter $p[n]$.

Following the above theories, FBMC waveform has various features, including low ISI, reduced OOB radiations, and low complexity due to PPN arrangement. Therefore, FBMC modulation has gained a lot of attention in wireless communication namely, $\mathrm{CR}$ and doubly-selective channel estimation errors at very high-speed scenario. ${ }^{18}$

However, few researchers have addressed the performance of FBMC in optical field. The aim of our work is to investigate the contribution of FBMC on WDM systems, especially when the channels spacing is reduced.

\section{Principle of SPM and XPM Computation}

It is well known that WDM technologies, with its different variants such as coarse WDM, dense WDM, and ultra-dense WDM, have been employed to increase the capacity of the optical 
channel. However, two broad categories of nonlinear phenomena arise: (i) intra-channel interference as SPM and (ii) ICI such as XPM and FWM. Their common factor is the severe dependence between the refractive index and the optical intensity, which is referred to as the Kerr effects. ${ }^{19}$ In contrast, ICI occurs because of the interaction among co-propagating channels. This dependence leads to a nonlinear phase shift in the optical field and inherent signal distortion. Here, we evaluate the impact of SPM and XPM over FBMC-WDM transmission, whereas the effects of FWM are neglected.

The evolution of the complex optical envelope $E_{i}(t, z)$ at the $i$ 'th-channel in a WDM system is modeled by the nonlinear Schrödinger equation (NLSE) which can be expressed as follows:

$$
\frac{\partial E_{i}}{\partial z}-j \frac{\beta_{2}}{2} \frac{\partial^{2} E_{i}}{\partial t^{2}}+\frac{\alpha}{2} E_{i}=-j \gamma_{i}\left(\left|E_{i}\right|^{2}+2 \sum_{\substack{k=1 \\ k \neq i}}^{N}\left|E_{k}\right|^{2}\right) E_{i},
$$

where $\beta_{2}$ is the group velocity dispersion (GVD) parameter, $\alpha$ is the fiber loss coefficient, $\gamma$ is the Kerr nonlinear coefficient, and the right-hand side of Eq. (3) accounts the SPM and XPM contributions. The NLSE equation cannot be solved analytically except for some special cases (i.e., for soliton solutions) ${ }^{20}{ }^{2}$ For this reason, it is of uttermost importance to appeal to another numerical approach. The most popular technique is the split-step Fourier method (SSFM) which consists of splitting the entire fiber length into sections of length $h$, and in each one, the electromagnetic field is subjected to linear and nonlinear operators. In the symmetric SSFM (S-SSFM), the nonlinear operator is encapsulated by two linear effects at the midpoint (i.e., $h / 2)$ as illustrated in Fig. 3. In other words, the dispersion effects are evaluated in frequency domain over $h / 2$, then, the signal is converted to the time domain to compute the nonlinear contributions along the entire section (i.e., $h$ ), and finally, the signal is converted back to the frequency domain to calculate the linear effects over the second half of the section.

For an accurate evaluation of XPM impacts, Leibrich and Rosenkranz ${ }^{21}$ proposed an efficient method with reduced complexity, which relies on the separate-channel approach applied to S-SSFM as defined in Algorithm 1.

Indeed, each channel in the WDM system is propagated independently, and the XPM contribution between $N$ channels is computed by the summation given in Eq. (3).

Assuming that the optical field $E_{i}$ is the output of the first linear step, the resulting field $E_{i}^{\prime}$ after accounting the nonlinear phase shift induced by SPM and XPM effects can be approximated in the time domain by

$$
E_{i}^{\prime}\left(t, z+\frac{h}{2}\right)=E_{i}\left(t, z+\frac{h}{2}\right) \exp (\overbrace{-j \gamma_{i} h \mid E_{i}\left(t, z+\left.\frac{h}{2}\right|^{2}\right.}^{\text {SPM }} \underbrace{-j 2 \gamma_{i} h \sum_{\substack{k=1 \\ k \neq i}}^{N} \mid E_{k}\left(t, z+\left.\frac{h}{2}\right|^{2}\right.}_{\text {XPM }}) \text {. }
$$

From Eq. (4), it is shown that the impact of XPM to the nonlinear phase shift dominates the SPM contribution in multichannel communication systems by a factor 2 due to the effects of the susceptibility of silica materials.

According to Ref. 21, the phase shift induced by XPM at the $i$ 'th channel can be accounted in the frequency domain by performing the fast Fourier transform (FFT) of the magnitude square of

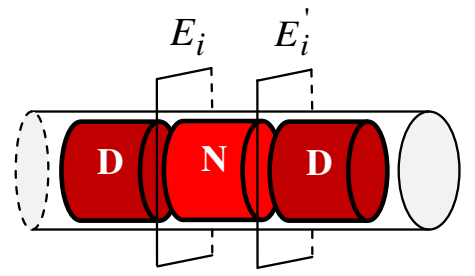

Fig. 3 Symmetric split-step Fourier methods: $D$ : linear operator; $N$ : nonlinear operator. 
Algorithm 1 SPM and XPM computation.



the neighboring channel $k$ and, then, applying a linear filter with frequency response $W_{i k}$ as expressed in Eq. (5):

$$
\begin{aligned}
\varphi_{i k}=2 \gamma_{i} \frac{1-e^{\left(j 2 \pi f d_{i k}-\alpha\right) h}}{\alpha-j 2 \pi f d_{i k}} \cdot e^{\left(\alpha-j 2 \pi d_{i k}\right) \frac{h}{2}} \\
. F F T\left(\left|E_{k}\left(t, z+\frac{h}{2}\right)\right|^{2}\right) \\
=W_{i k} \cdot F F T\left(\left|E_{k}\left(t, z+\frac{h}{2}\right)\right|^{2}\right),
\end{aligned}
$$

where $d_{i k}$ is the walk-off corresponding to the group velocity mismatch between the channel of interest $i$ and channel $k$. The group velocity is defined at nonzero dispersion band as ${ }^{22}$

$$
d_{i k}=\int_{\lambda_{k}}^{\lambda_{i}} D(\lambda) \mathrm{d} \lambda \quad \approx D\left(\lambda_{i}-\lambda_{k}\right),
$$

where $D$ is the dispersion parameter of the fiber.A detailed pseudocode and flowchart summarizing the proposed algorithm are given in Figs. 4 and 5, respectively.

\section{SPM and XPM Post-Compensation Using Volterra Equalizer}

The Volterra series can be assimilated to a Taylor series with memory in terms of nonlinear modeling. ${ }^{23}$ The implementation of Volterra equalizer embodies linear and nonlinear filters, which makes it a powerful tool to compensate both linear and nonlinear effects. In a discrete time, causal nonlinear system, the relation between the input $x(n)$ and output sequence $Y(n)$ is defined as ${ }^{24}$ 
Gharbi, Mhatli, and Attia: Cross-phase modulation compensation in multichannel system based...



Fig. 4 Flowchart of SPM and XPM contribution estimation at midpoint section.

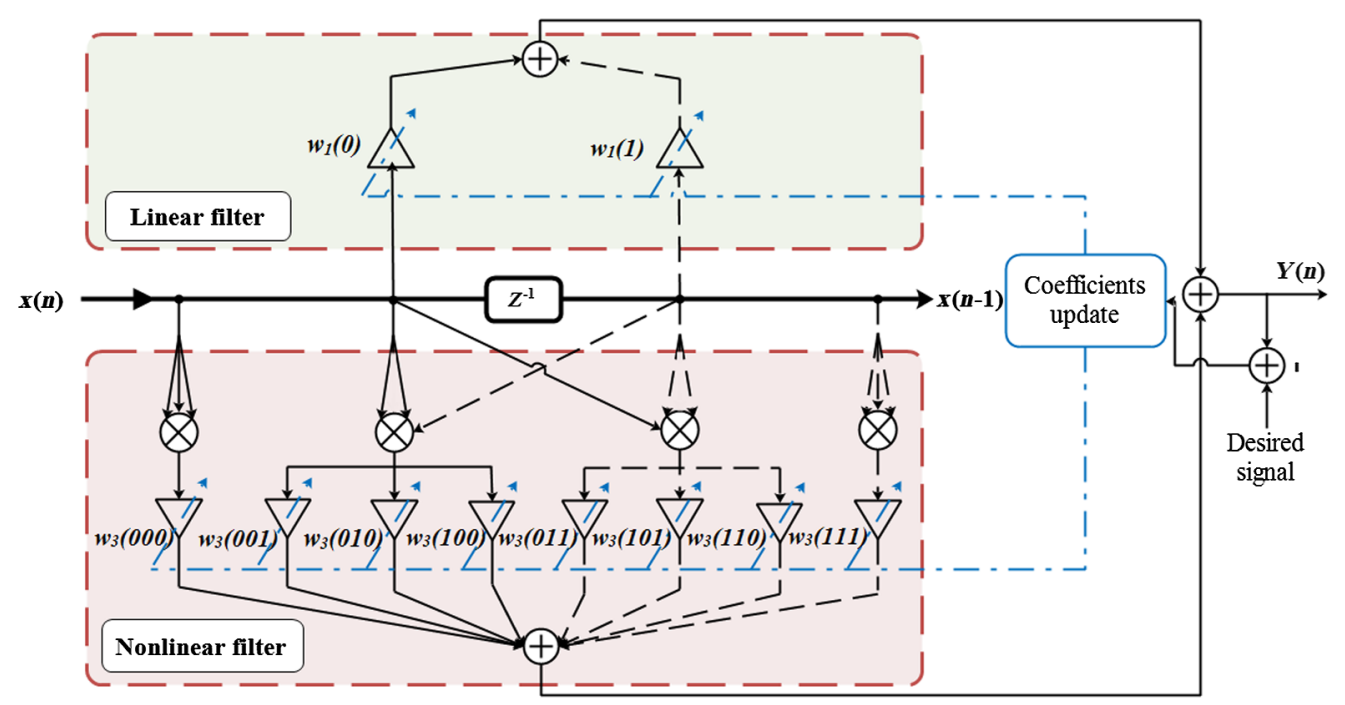

Fig. 5 Implementation of TOV with $M=2$. 


$$
\begin{aligned}
Y(n)= & w_{0}+\sum_{i_{1}=0}^{\infty} w_{1}\left(i_{1}\right) x\left(n-i_{1}\right)+\sum_{i_{1}=0}^{\infty} \sum_{i_{2}=0}^{\infty} w_{2}\left(i_{1}, i_{2}\right) x\left(n-i_{1}\right) x\left(n-i_{2}\right)+\ldots \\
& \sum_{i_{1}=0}^{\infty} \ldots \sum_{i_{j}=0}^{\infty} w_{j}\left(i_{1} \ldots i_{j}\right) x\left(n-i_{1}\right) \ldots x\left(n-i_{j}\right),
\end{aligned}
$$

where $w_{0}$ is a constant and $w_{j}().(j \neq 0)$ is the $j$ 'th-order kernels of the Volterra model.

The computational complexity of the full Volterra series explodes exponentially and cannot be implemented in real applications. ${ }^{25}$ Therefore, the higher-order terms of full Volterra are dismissed and usually truncated by limiting the order of the series and taking into account the tradeoff between complexity and performance. In practice, the third-order Volterra (TOV) is sufficient to counter the nonlinear effects and the input-output relation is given as

$$
Y(n)=\sum_{i_{1}=0}^{M} w_{1}\left(i_{1}\right) X\left(n-i_{1}\right)+\sum_{i_{1}=0}^{M-1} \sum_{i_{2}=0}^{M-1} \sum_{i_{3}=0}^{M-1} w_{3}\left(i_{1}, i_{2}, i_{3}\right) X\left(n-i_{1}\right) X\left(n-i_{2}\right) X\left(n-i_{3}\right) .
$$

In Eq. (8), we assume that $w_{0}=0$ and $M$ represents the memory length. It is worth mentioning that in bandpass systems, the even-order kernels are ignored and only odd-order terms are retained. ${ }^{26}$ Many algorithms are used to recursively adjust the coefficients of the filter, the most popular are least mean square (LMS) and recursive least square (RLS). In our work, the RLS algorithm is performed to ensure a rapid convergence toward the optimum solutions. The goal of this method is to minimize the error function given as the sum of the difference between the desired signal and the estimated output for all the samples. ${ }^{27}$

The structure of the adaptive TOV equalizer with a memory of two $(M=2)$ is depicted in Fig. 5.

\section{System Setup}

Commercial MATLAB software is used to implement the proposed architecture as shown in Fig. 6.

In our simulation, six channels WDM are considered and the corresponding data rate is $120 \mathrm{~Gb} / \mathrm{s}$ (i.e., $6 \times 20 \mathrm{~Gb} / \mathrm{s}$ ). Each channel conveys PPN-FBMC/OQAM signal, where the total number of subcarriers is 512. The real and imaginary components of the transmitted signals are injected into digital-to-analog converters (DACs). Next, the resulting signals are converted to the optical domain using an $I / Q$ modulator. To ensure that the MZM modulators operate in the linear modulation region, a DC bias voltage is applied. The optical signals are launched over $100 \mathrm{~km} \times 30$ spans of SMF fiber, which is modeled by S-SSFM. An erbium-doped fiber amplifier (EDFA) is implemented to amplify the distorted optical signal. A coherent receiver with a local oscillator is used to properly track the phase and the amplitude of the signals.



Fig. 6 Simulation setup for a coherently detected WDM system. DAC, digital-to-analog converter; LPF, low-pass filter; LD, laser diode; MZM, Mach-Zehnder modulator; MUX, multiplexer; SMF, single-mode fiber; EDFA, erbium-doped fiber amplifier; DEMUX, de-multiplexer; PD, photodiode; $A D C$, analog-to-digital converter. 
Table 1 Transmitter configuration.

\begin{tabular}{lc}
\hline \hline Parameter & Value \\
\hline Number of subcarriers $(N)$ & 512 \\
Modulation & $16-O Q A M$ \\
Prototype filter & PHYDYAS \\
Overlapping factor $(k)$ & 4 \\
Sampling frequency $(F s)$ & $5 \mathrm{GHz}$ \\
Data rate/channel & $20 \mathrm{~Gb} / \mathrm{s}$ \\
\hline \hline
\end{tabular}

Table 2 SMF parameters.

\begin{tabular}{lc}
\hline \hline Parameter & \multicolumn{1}{c}{ Value } \\
\hline Attenuation $(\alpha)$ & $0.2 \mathrm{~dB} / \mathrm{Km}$ \\
GVD $\left(\beta_{2}\right)$ & $-21.7 \mathrm{e}^{-27} \mathrm{~s}^{2} / \mathrm{m}$ \\
Nonlinear refractive index $\left(n_{2}\right)$ & $3.10^{-20} \mathrm{~m}^{2} / \mathrm{W}$ \\
Effective area $\left(A_{\text {eff }}\right)$ & $80 \mu \mathrm{m}^{2}$ \\
Dispersion $(D)$ & $17 \mathrm{ps} / \mathrm{nm} \mathrm{km}$ \\
Span length & $100 \mathrm{~km}$ \\
Number of spans & 30 \\
Central wavelength $(\lambda)$ & $1.53455 \mathrm{~nm}$ \\
Channel spacing & $12.5,25,50,100 \mathrm{GHz}$ \\
Laser linewidth & $100 \mathrm{kHz}$ \\
\hline \hline
\end{tabular}

The transmitted signals are generated with the parameters reported in Table 1, whereas Table 2 illustrates the characteristic of the optical channel.

\section{Results and Discussions}

It is well known that the impact of XPM is deeply related to the channel spacing and the operating bit rates. Moreover, the nonlinear effects increase with the intensity of the launched optical signal, which can be countered using new fibers with a large effective area.

In this context, the following results investigate the central PPN-FBMC/OQAM channel (i.e., third channel) over $3000 \mathrm{~km}$ of SMF in terms of these three key factors.

\subsection{Impact of Channel Spacing}

Figure 7 shows the bit error rate (BER) performance as a function of the launched optical power for the central channel spaced by $100,50,25$, and $12.5 \mathrm{GHz}$, which is equivalent to $0.8,0.4,0.2$, and $0.1 \mathrm{~nm}$, respectively. A 3-taps LMS filter is employed as a linear filter and compared with 3-taps TOV.

We observe that the impact of XPM is inversely proportional to the channel spacing and it can be ignored for wide channel spacing, $100 \mathrm{GHZ}$ and higher. ${ }^{28}$ The optimum optical powers (i.e., inflection points) are obtained at $-10 \mathrm{dBm}$ for 100 and $50 \mathrm{GHz}$ and $-12 \mathrm{dBm}$ for 25 and 


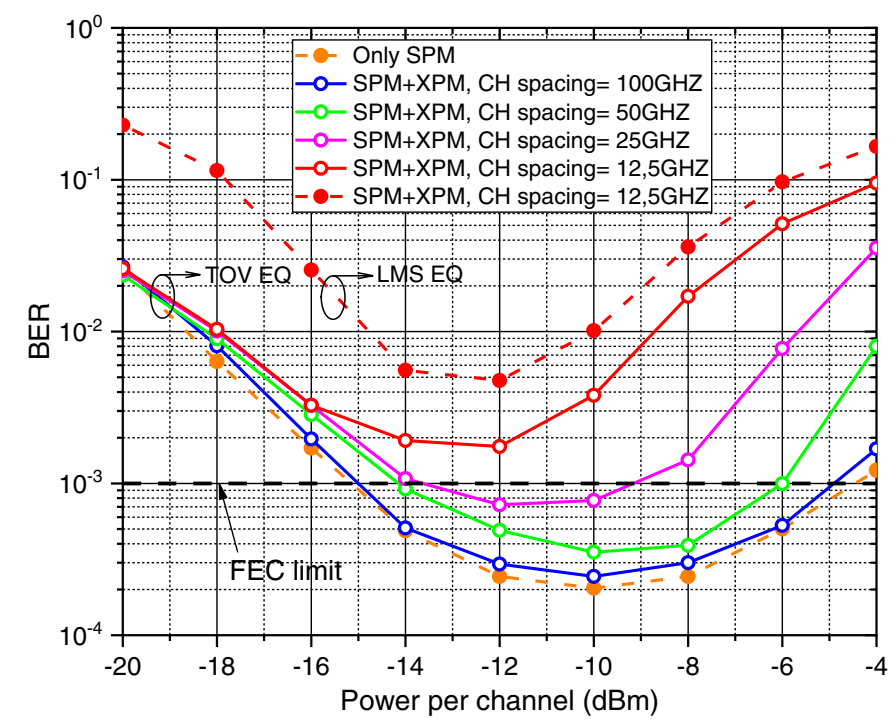

Fig. 7 BER versus launched optical power for different channel spacing using linear (solid circle) and TOV (empty circle) equalizers.

12.5 GHz channel spacing, which means that the performance of the channel becomes more sensitive to the launched power for closest channels.

Indeed, both SPM and XPM distortions alter the phase of the signal, which experience a broadening of the pulse spectrum. For reduced channel spacing, the adjacent channels overlap in the time domain and significantly affect the performance of the system. In other words, the XPM crosstalk is inversely proportional to the walk-off rate (i.e., $d_{i k}$ ) and this distortion is reduced for larger channel spacing. It should be noticed that the channel spacing has no effects on SPM.

At 12.5-GHz channel spacing, the LMS filter fails to meet the performance of the TOV filter when the same number of taps is used. However, the number of taps needs to be increased for the TOV equalizer to reach the BER threshold before forwarding error correction given at $10^{-3}$.

The second set of analysis assesses the impact of the number of taps for TOV equalizer at 12.5-GHZ spacing. For convenience, the $Q$ factor is used as a quality metric, which is related to BER by

$$
Q(\mathrm{~dB})=20 \log _{10}\left(\sqrt{2} \operatorname{erfc}^{-1}(2 * \mathrm{BER})\right) .
$$

Figure 8 shows that a $Q$-factor of $9.8 \mathrm{~dB}$, which corresponds to $\mathrm{BER}=10^{-3}$, can be reached at $-12 \mathrm{dBm}$ when using TOV filter with 5 taps. By increasing the number of taps from 3 to 7 , an improvement of approximately $1 \mathrm{~dB}$ can be obtained. The increase of taps number leads to an expansion of the memory length. Hence, the distortions are accurately compensated. The complexity and the number of required coefficients for TOV equalizer are discussed later.

The enhancement in system performance in terms of constellation diagrams for different taps number at $-12 \mathrm{dBm}$ input power is shown in Fig. 9.

\subsection{Impact of Bit Rates}

Unlike FWM effects, XPM is mainly dependent on the operating bit rates. The results in Fig. 10 outline the influence of bit rate for $12.5-\mathrm{GHz}$ channel spacing by varying the number of taps of the proposed filter.

The computer simulations show that the increase of the bit rate inherently degrades our system performance and a bit rate of $22.5 \mathrm{~Gb} / \mathrm{s}$ per channel is tolerated when 7-taps TOV equalizer is used. Indeed, XPM distortion at high bit rates gives rise to a multipeak spectrum due to the 
Gharbi, Mhatli, and Attia: Cross-phase modulation compensation in multichannel system based...

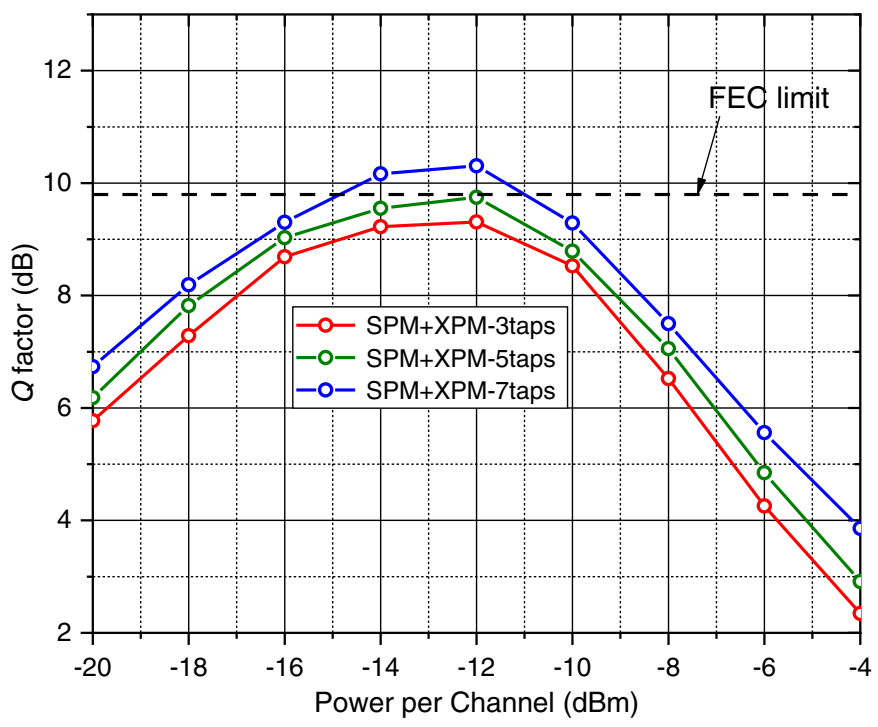

Fig. $8 Q$-factor versus launched optical power for different number of taps of TOV filters at 12.5-GHZ channel spacing.

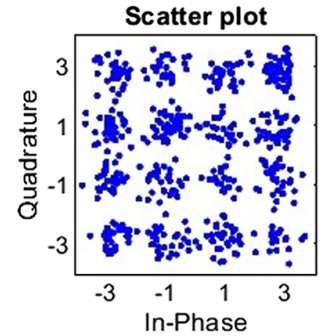

(a)

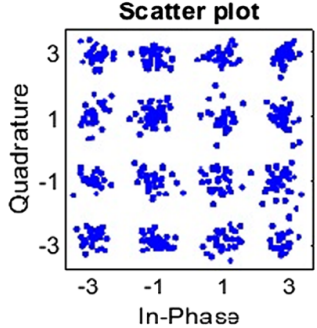

(b)



(c)

Fig. 9 Constellation diagram for (a) 3 taps, (b) 5 taps, and (c) 7 taps of TOV equalizer with 12.5-GHZ channel spacing.

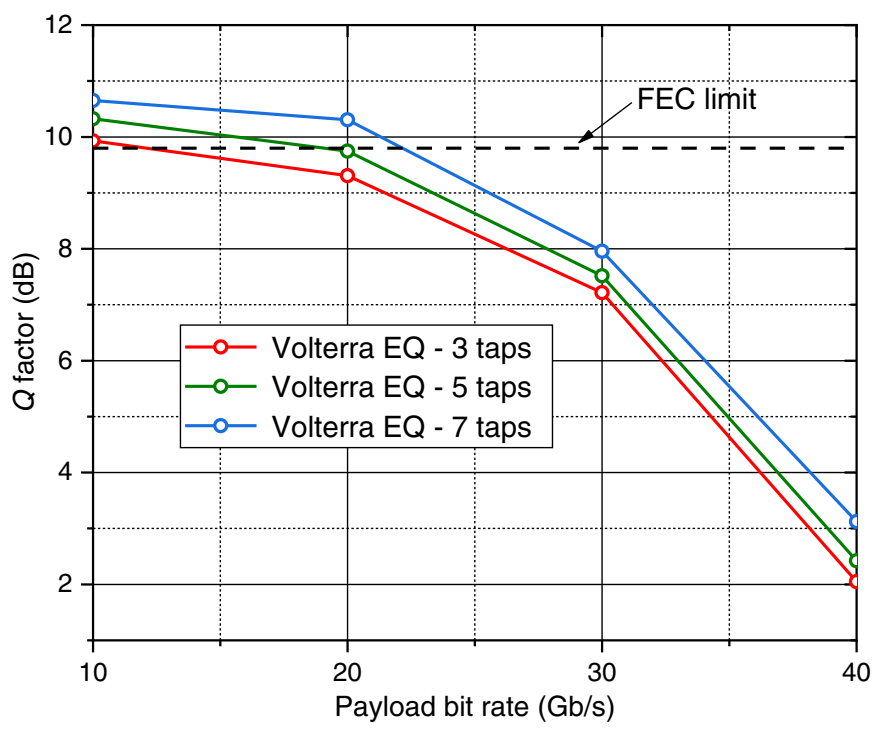

Fig. $10 Q$-factor versus payload bit rate for different taps of TOV filter. 
increased number of transitions in the time domain, which spread out the spectrum. Thus, interference between adjacent peaks occurs.

\subsection{Impact of Fiber Types}

The nonlinear effects are inversely proportional to the effective area of the fiber $\left(A_{\text {eff }}\right)$. Among the proposed solution to improve the fiber linearity is the deployment of a new class of fibers with a large effective area, namely, pure-silica-core fiber (PSCF). ${ }^{29}$ Figure 11 shows the performance of three types of optical fiber, including non-zero dispersion-shifted fiber (NZDSF), standard-SMF, and PSCF. The physical specifications of these fibers are given in Table 3.

At $12.5-\mathrm{GHz}$ channel spacing, the system with PSCF fiber outperforms the other simulated fiber due to the lower Kerr nonlinear parameter $\gamma$ resulted in the larger effective area $(60 \%$ higher than SSMF). As illustrated in Fig. 11, the optimal input power range is defined from -16 to $-8 \mathrm{dBm}$. Since the nonlinear parameter increases with the decrease of the effective area, the nonlinear effects are more pronounced for NZDSF fiber and it becomes more sensitive to optical intensity higher than $-16 \mathrm{dBm}$. Moreover, the transition to the nonlinear regime occurs at $12 \mathrm{dBm}$ when SSMF fiber is used, whereas the PSCF fiber allows higher optical power in the vicinity of $-8 \mathrm{dBm}$ of launched power. Indeed, fibers with large effective area relax the light confinement in the core and decrease the power density. ${ }^{30}$

As shown in Fig. 12, NZDSF fiber shown its limits to reach the required performance $(Q=9.8 \mathrm{~dB})$ even for 5 taps and when compared to SSMF fiber, PSCF fiber gives an improvement of almost 1.3 and $1 \mathrm{~dB}$ for 3 and 5 taps, respectively.

One can note that the Raman amplification technique cannot be exploited when fibers with large effective area are used because it requires a very high intensity of power.

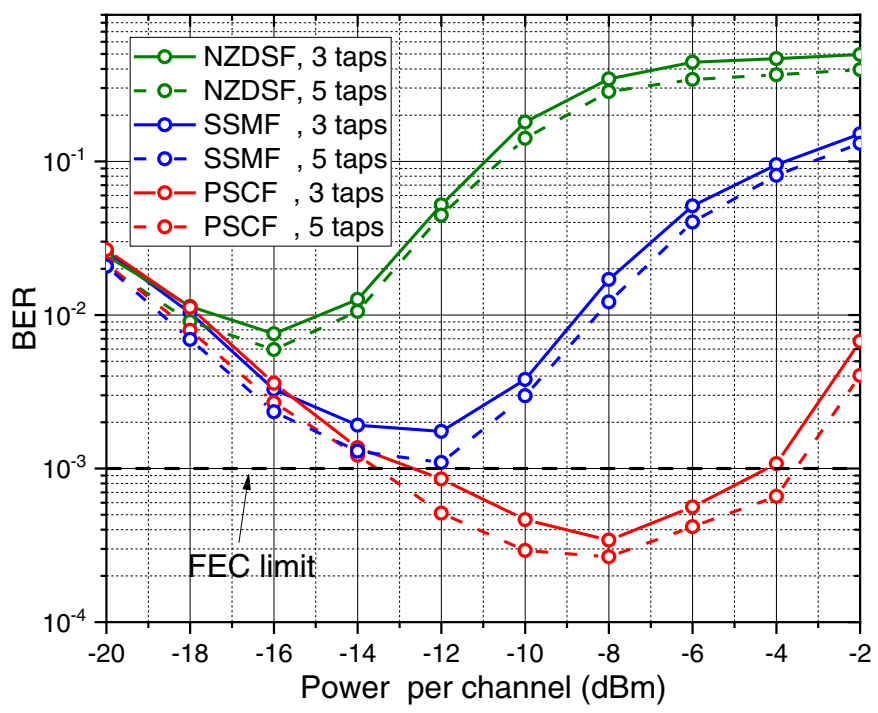

Fig. 11 BER versus launched optical power for different fiber types and taps number.

Table 3 Physical specifications of different fiber types.

\begin{tabular}{lccc}
\hline \hline Parameter & NZDSF & SSMF & PSCF \\
\hline$\alpha(\mathrm{dB} / \mathrm{km})$ & 0.19 & 0.2 & 0.16 \\
$D(\mathrm{ps} / \mathrm{nm}-\mathrm{km})$ & 4 & 17 & 21 \\
$A_{\text {eff }}\left(\mu \mathrm{m}^{2}\right)$ & 70 & 80 & 130 \\
$\gamma\left(\mathrm{km}^{-1} \mathrm{~W}^{-1}\right)$ & 1.8 & 1.5 & 0.94 \\
\hline \hline
\end{tabular}


Gharbi, Mhatli, and Attia: Cross-phase modulation compensation in multichannel system based...

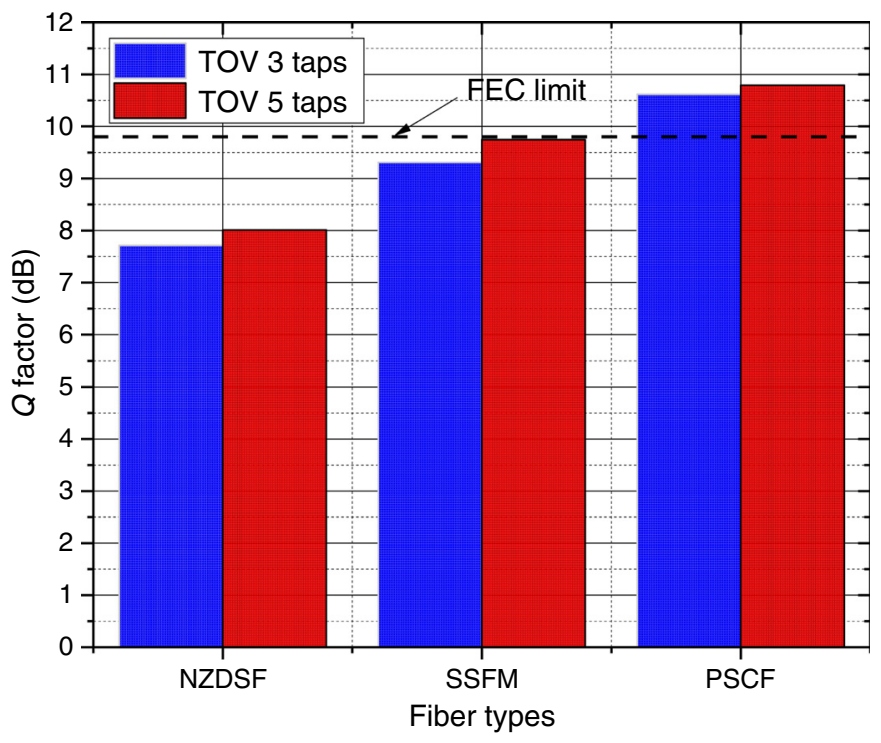

Fig. $12 Q$-factor versus launched optical power for NZDSF, SSMF, and PSCF at their optimum launched power $-16 \mathrm{dBm},-12 \mathrm{dBm}$, and $-8 \mathrm{dBm}$, respectively.

\section{Volterra Filter Complexity}

The total number of multiplications required for the $P$ 'th order Volterra filter with memory length $M$ is expressed in Eq. (10) and depicted in Fig. 13:

$$
C_{\text {VOlterra }}(P, M)=\sum_{\substack{p=1 \\ p \text { odd }}}^{P} \frac{(M-1+p) !}{(p-1) ! \times(M-1) !} .
$$

Figure 13 shows that the computational complexity of the Volterra filter is strongly linked to the order of the series $P$ and the memory length $M$. In other words, the number of multiplications escalates with the increase of the nonlinearity order and the memory depth. For simplicity, the Volterra series is truncated to cubic order, which ensures an acceptable performance with a minimum of complexity. The number of Volterra kernel (i.e., coefficient) needed for the filtering process is $^{15}$

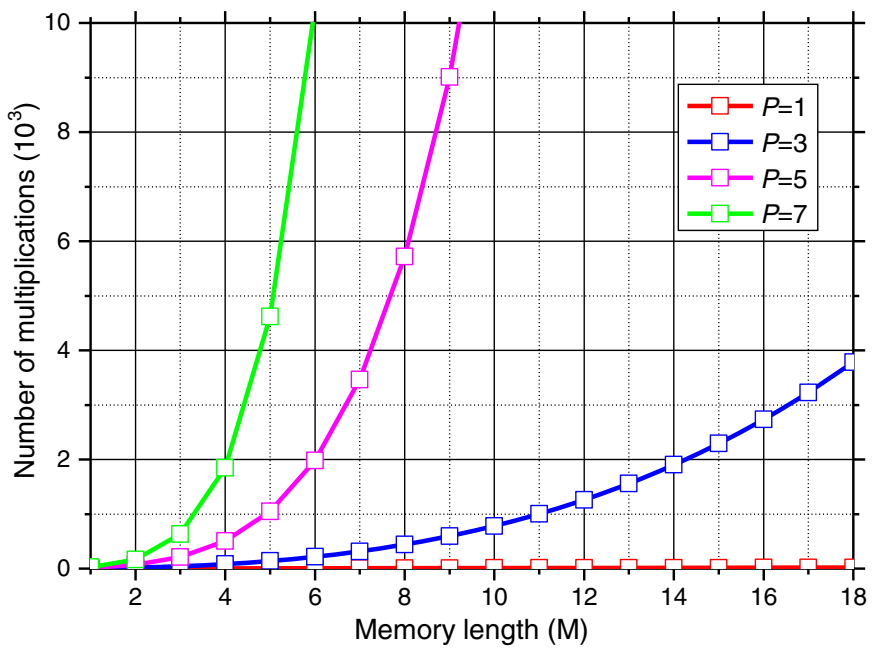

Fig. 13 The computational complexity of Volterra filter for various order $P$ when varying the memory length $M$. 


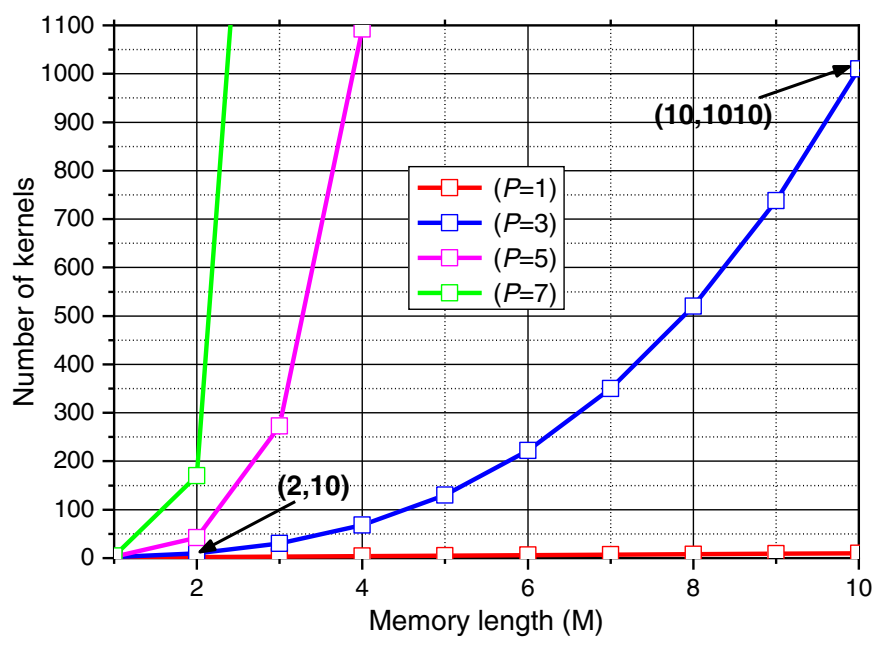

Fig. 14 Kernel number versus memory length for different nonlinearity order.

$$
K_{\text {VOLTERRA }}(P, M)=\sum_{\substack{p=1 \\ p \text { odd }}}^{\frac{P+1}{2}} M^{2 p-1} .
$$

From Fig. 14, it can be seen that the number of coefficients increases exponentially with the nonlinear order and the memory length, which poses a big challenge for systems with high nonlinearities. For example, TOV with the memory of two $(M=2)$ requires 10 coefficients (as illustrated in Figs. 5 and 14), whereas increasing the memory length by eight (i.e., $M=10$ ), the number of kernel increases more than one thousand.

Hence, there is a compromise to be made between performance by employing higher-order and computational complexity of the system.

\section{Conclusion}

FBMC-OQAM is a promising technology for B5G networks due to its efficient spectrum utilization and robustness against channel distortions. On the other hand, new flexible air interface and innovative scheme-based fiber technology are under consideration.

In this paper, we propose, for the first time, a new longhaul system based on FBMC/OQAM waveform in WDM link. XPM and SPM distortions are investigated for $6 \times 20 \mathrm{~Gb} / \mathrm{s}-\mathrm{WDM}$ scheme over $3000 \mathrm{~km}$, and Volterra equalizer is proposed to compensate the channel nonlinearity impairments. Analytical analysis and simulations results show that FBMC/OQAM with PPN is an appealing scheme for longhaul WDM link with reduced channel spacing.

The results show that an improvement of $1 \mathrm{~dB}$ is obtained for 7-taps TOV filter compared with the same filter using 3 taps at 12.5-GHZ channel spacing. Compared to SSMF fiber, PSCF with large effective is suitable in some cases and exhibits a gain of approximately 1.3 and $1 \mathrm{~dB}$ for 3 and 5 taps, respectively.

\section{References}

1. L. Y. Wei et al., "Tricolor visible-light laser diodes based visible light communication operated at $40.665 \mathrm{Gbit} / \mathrm{s}$ and $2 \mathrm{~m}$ free-space transmission," Opt. Express 27(18), 25072-25077 (2019).

2. R. Zhang et al., "An ultra-reliable MMW/FSO A-RoF system based on coordinated mapping and combining technique for $5 \mathrm{G}$ and beyond mobile fronthaul," J. Lightwave Technol. 36(20), 4952-4959 (2018).

3. M. A. Hasabelnaby, H. A. I. Selmy, and M. I. Dessouky, "Joint optimal transceiver placement and resource allocation schemes for redirected cooperative hybrid $\mathrm{FSO} / \mathrm{mmW} \mathrm{5G}$ fronthaul networks," J. Opt. Commun. Networking 10(12), 975-990 (2018). 
Gharbi, Mhatli, and Attia: Cross-phase modulation compensation in multichannel system based...

4. D. Feng, W. Sun, and W. Hu, "CAPEX optimization with joint allocation of hybrid RF/FSO and optical fibre resources in 5G backhaul," Opt. Fiber Commun. Conf., OSA Technical Digest (Online), Optical Society of America (2018).

5. N. S. Bergano, "Wavelength division multiplexing in longhaul transoceanic transmission systems," J. Lightwave Technol. 23, 4125-4139 (2005).

6. I. Morita, "Ultra-dense WDM transmission technologies toward $100 \%$ spectral efficiency," in Proc. Eur. Conf. Opt. Commun., Rimini (2003).

7. A. Omri et al., "Synchronization procedure in 5G NR systems, IEEE Access 7, 4128641295 (2019).

8. T. Jiang et al., OQAM/FBMC for Future Wireless Communications: Principles, Technologies, and Applications, Elsevier, Academic Press (2018).

9. H. Song et al., "Combined constrained code and LDPC code for longhaul fiber-optic communication systems," in IEEE Global Commun. Conf. (GLOBECOM) (2012).

10. L. Liu et al., "Performance evaluation of high-speed polar coded CO-OFDM system with nonlinear and linear impairments," IEEE Photonics J. 9(4), 7202909 (2017).

11. S. T. Le et al., "Optical and digital phase conjugation techniques for fiber nonlinearity compensation," in Opto-Electron. and Commun. Conf. (OECC) (2015).

12. G. P. Agrawal, Fiber-Optic Communication Systems, 3rd ed., Wiley, John \& Sons (2002).

13. R. Dar et al., "Inter-channel nonlinear interference noise in WDM systems: modeling and mitigation," J. Lightwave Technol. 33(5), 1044-1053 (2015).

14. R. Nissel, S. Schwarz, and M. Rupp, "Filter bank multicarrier modulation schemes for future mobile communications," IEEE J. Sel. Areas Commun. 35(8), 1768-1782 (2017).

15. A. Viholainen et al., "Prototype filter design for filter bank based multicarrier transmission," in 17th Eur. Signal Process. Conf. (EUSIPCO 2009), Glasgow (2009).

16. M. Bellanger et al., "FBMC physical layer: a primer," PHYDYAS (2010).

17. M. Tanda et al., "Data-aided synchronization and initialization (single antenna), PHYDYAS: physical layer for dynamic access and cognitive radio," Deliverable D2.1 (2008).

18. M. Saideh, I. Dayoub, and M. Berbineau, "Efficient equalization for FBMC-OQAM under doubly selective channel estimation errors," IEEE Commun. Lett. 23(5), 863-866 (2019).

19. G. P. Agrawal, Nonlinear Fiber Optics, 4th ed., Academic Press (2007).

20. E. Forestieri and M. Secondini, "Solving the nonlinear Schrödinger equation," in Optical Communication Theory and Techniques, E. Forestieri, Ed., pp. 3-11, Springer US, Boston, Massachusetts (2005).

21. J. Leibrich and W. Rosenkranz, "Efficient numerical simulation of multichannel WDM transmission systems limited by XPM," IEEE Photonics Technol. Lett. 15(3) (2003).

22. T. Chiang et al., "Cross-phase modulation in fiber links with multiple optical amplifiers and dispersion compensators," J. Lightwave Technol. 14, 249-260 (1996).

23. D. W. Liu, J. C. Principe, and S. Haykin, Kernel Adaptive Filtering: A Comprehensive Introduction, p. 24, Wiley (2011).

24. J. Pan and C.-H. Cheng, "Nonlinear electrical compensation for the coherent optical OFDM system," J. Lightwave Technol. 29(2), 215-221 (2011).

25. V. J. Mathews, "Adaptive polynomial filters," IEEE Signal Process. Mag. 8(3), 10-26 (1991).

26. A. Gutierrez and W. E. Ryan, "Performance of Volterra and MLSD receivers for nonlinear band-limited satellite systems," IEEE Trans. Commun. 48(7), 1171-1177 (2000).

27. O. Gharbi et al., "Numerical investigation of longhaul coherent optical generalized frequency division multiplexing signal," IEEE Photonics J. 11, 7203514 (2019).

28. Y. Yamamoto, M. Hirano, and G. Sasaki, "A new class of optical fiber to support large capacity transmission," in Opt. Fiber Commun. Conf./Nat. Fiber Opt. Eng. Conf., 3rd ed., Morgan Kaufmann Publishers, Burlington, Massachusetts (2011).

29. Y. Yamamoto, M. Hirano, and Sasaki Takashi, "A new class of optical fiber to support largecapacity transmission," in Opt. Fiber Commun. Conf. (2011).

30. K. S. Mathur, Fundamentals of Fiber Optics Communications, Zorba Books (2018). 
Oussama Gharbi received his telecommunication engineer degree from the Ministry of National Defense of Tunisia in 2010 and he joined the Electronic Systems and Communications Networks Laboratory (SERCOM) in the Polytechnic School of Tunisia. His current research interests include optical communications, FTTX, X-PON, X-WDM, fiber non-linearities compensation, $5 \mathrm{G}$ and beyond waveform design, and multicarrier systems.

Sofien Mhatli is a senior researcher at Ecole Polytechnique de Tunis and a telecommunications engineer at the Ministry of National Defense of Tunisia. His expertise is focused on designing and optimizing optical and microwave devices for applications in wireless telecom, sensing systems, and optical communication systems (SDH, WDM, PON, CO-OFDM, IM-DD, and so on). He has deep knowledge in digital signal processing, algorithmic design (e.g., machine learning), and optical devices (e.g., MZM) for optical communications. The outcome of his research resulted in prestigious peer-reviewed journal papers and top conference presentations (e.g., OSA Optics Letters, OFC, IEEE Photonics Journal, and ECOC).

Rabah Attia received his $\mathrm{PhD}$ in telecommunication from the University of Valenciennes, France, in 1986. He is currently a professor of optical communication at the Tunisia Polytechnic School (TPS). Since 2004, he is co-responsible for the System and Component Electronic Research Lab at the TPS. His main research interest includes the new generation of optical fiber, photonic crystal component, and electro-optic modulators. 